\title{
Meraque or Triple Energizer (San Jiao): Actual or Virtual Organ in Traditional Medicine - A Hypothetical Viewpoint
}

\author{
Majid Avijgana Mahtab Avijgan ${ }^{b}$ \\ ${ }^{a}$ Department of Traditional Persian-Iranian Medicine, Isfahan University of Medical \\ Sciences, Isfahan, Iran; b The University of Newcastle, Newcastle, N.S.W., Australia
}

\section{Key Words}

Anatomy $\cdot$ Coelom $\cdot$ Embryology $\cdot$ Iranian traditional medicine $\cdot$ Meraque $\cdot$ San Jiao $\cdot$

Traditional Chinese medicine · Triple Energizer · Primo-vascular system

\begin{abstract}
Background/Objective: In traditional medicine, there are a number of terms and organs that have not yet been reported in Western medicine. For example, 'Meraque' in Iranian traditional medicine and 'Triple Energizer' or 'San Jiao' in traditional Chinese medicine (as one of the Fu organs) are examples of such terms. The organs they refer to can explain many of the clinical signs and symptoms which have been noted as psychosomatic disorders in Western medicine. One hypothesis is that this organ might be a real space. From the viewpoint of embryology and anatomy, this report will try to create a new perception in the reader's mind of the Meraque/Triple Energizer/San Jiao of the human body. Methods: We searched through the major databases for the key words 'traditional medicine', 'Meraque', 'meridians', 'acupuncture points', 'San Jiao' and 'Triple Energizer'. Results: There were no reports on the relevant concepts of San Jiao/Triple Energizer and Meraque. Discussion: During embryonic development and after gastrulation, three layers called the ectoderm, mesoderm and endoderm are formed. The mesoderm is the source of most of the internal organs, dividing into two layers, the somatic and the visceral, with an invisible space in between that is called the coelom. This space could possibly be the same as the Meraque/Triple Energizer/San Jiao (as a hollow organ) and, furthermore, the same as a cavitary tissue forming a pathway to connect the internal organs of the body with the surface of the body. In this case, many of the mechanisms of traditional
\end{abstract}

Some parts of this paper were presented at the 2 nd International Conference on Traditional and Alternative Medicine, Beijing, 2014. 
medicine and acupuncture can be explained. In other words, for the first time the coelom is proposed as the Meraque and the Triple Energizer/San Jiao in Iranian and Chinese traditional medicine, respectively.

(C) 2015 S. Karger AG, Basel

\section{Introduction}

In the Tebbe Akbari, one of the Iranian traditional medicine (ITM) sources written 320 years ago (which is a Persian translation of an old source of ITM written 820 years ago, the Asbab va Alaem), Hakim Mohammad Akbar Arzani explained the Meraque in chapter 20. Also, there is a space or paths called Ghalaz vessels which may be equivalent to acupuncture channels [1] and/or the Meraque space, although there might be two different items or interstitial spaces in ITM.

On the other hand, in traditional Chinese medicine (TCM), the basic concept includes the theory of Zang-Fu organs with five Zang (Yin) organs. Yin organs (Zang) are solid organs, i.e. the Heart, Spleen, Lung, Kidney and Liver; each one of them is paired with a hollow or Fu organ, i.e. the Small Intestine, Stomach, Large Intestine, Urinary Bladder and Gall Bladder, respectively. The sixth organ of Fu, the Triple Energizer (TE) or San Jiao (SJ), is unknown in all variants of traditional and nontraditional medicine except for TCM. Yet, no report has anatomically discussed the TE/SJ as a true space, except for the fact that it is considered as a body cavity in some of the old textbooks of TCM [2-4].

It seems that the Meraque and the TE/SJ are based on similar spatial concepts in ITM and TCM, respectively. This cavity or space is introduced as an organ, tissue or place which influences other organs of the body. This report will try to revise the views regarding anatomical and embryonic organs or tissues in order to explain the Meraque/TE/SJ. This is the first time that the Meraque/TE/SJ is interpreted as a hollow organ or cavity (Fu organ in TCM) in both traditional and biomedicine (modern medicine). Some parts of this report were presented at the 2nd International Conference on Traditional and Alternative Medicine, Beijing, 2014 [5]. Here we present the complete report and hypothesis.

\section{Materials and Methods}

The literature was reviewed looking for 9 key words/themes: 'organogenesis', 'embryogenesis', 'anatomy', 'traditional Chinese medicine/TCM', 'traditional medicine', 'Meraque', 'San Jiao', 'Triple Energizer' and 'Triple Burner'.

\section{Search Strategy}

The following databases were searched for peer-reviewed articles in English: Cochrane Database, PubMed, MEDLINE, CINAHL, AMED, AgeLine and Social Services Abstracts. The following databases were searched for articles in Persian (Farsi): Iranmedex, Daneshyar and magiran for papers on the Meraque.

Our search included the following key words individually or in combination: alternative medicine(s), integrated medicine, holistic care, complementary alternative medicine, embryology, traditional Chinese medicine and traditional medicine, in relation to San Jiao, Triple Burner, Triple Energizer and Meraque. There were no reports explaining the implications of the SJ, Triple Burner, TE or Meraque in anatomy and embryogenesis. Our hypothesis is necessarily to be proven by the developmental periods of human embryogenesis presented in the following, accompanied by pictures or figures of organogenesis or embryonic life. The 
Meraque/TE/SJ is a broad network in the human body; it is suggested to be a true but invisible structure connecting all organs of the body with each other to produce a whole. This organ, which is distributed in surface organs and between organs and tissue, is the connective tissue.

\section{Results and Discussion}

To understand the theory of Meraque and TE/SJ, the mesoderm as one of the fetal layers should be discussed. As shown in embryonic development, while gastrulation occurs, a single layer of blastula will differentiate into the trilaminar blastoderm or three layers of ectoderm, mesoderm and endoderm [6].

In our previous studies, details can be found about the theory of the primo-vascular system (PVS) [7] and also about the origin of the PVS [8]. The PVS is a new, primitive circulatory system which is reported to regulate the embryonic developmental process [8] and is composed of ducts, corpuscles and granules [7]. Each granule (sanal) is floating in ducts, undergoes a 'sanalization' process (a process by which it grows into cells and becomes a granule) and is composed of a 'sanalsome' (containing DNA) and 'sanalplasm' (containing RNA). It plays a crucial role in physiological functions of the PVS (such as dead cell regeneration) [7].

During the sanalization process, the number of sanalsomes stays the same as the number of chromosomes. Primo-microcells move into primo-vessels, then grow, mature, migrate to local tissue and differentiate into stem cells [6]. In the PVS, primo-microcells control organogenesis, grow and differentiate into pluripotent stem cells, which are undifferentiated cells or very small embryonic-like stem cells, deposited as a messenger in organs during organogenesis and gastrulation. Epiblast or germ layer stem cells derive from pluripotent stem cells and serve as reserve cells, i.e. as inactive pluripotent or adult stem cells [7].

The granules are precursors of the process of inner cell mass transformation into the epiblast. The PVS controls differentiation of the epiblast into the ectoderm (giving rise to the skin and central nervous system), mesoderm (giving rise to the mesenchymal cells) and endoderm (giving rise to the endothelial cells of the digestive and respiratory system, and so on) [7].

The PVS appears before or simultaneously with fertilization, during which two things form: one polar body (which transforms into the PVS later on) and two pronuclei. The chromosomes in the first polar body are the same as in the oocyte after first meiotic division. The transformation of the polar body into the PVS happens in a space between the zona pellucida and the zygote. The PVS is responsible for the early cell differentiation, cell junction and cavitation process [8].

In other words, there is a close relationship between and a controlling effect of the three embryonic layers in the process of embryonic development into a fetus. The ectoderm includes the extraembryonic PVS [8], and BMP4 secreted by the ectoderm (originally the PVS) triggers mesoderm formation or differentiation (by intercellular signaling) [9]; then, the mesoderm transforms into polarized tissue and acts as an organizer center [10]. Based on our theory, this process could be stimulated by the PVS or extraembryonic PVS of the ectoderm [8].

When the embryo evolves, the mesoderm begins to split into three main areas: the paraxial mesoderm, the intermediate mesoderm and the lateral plate mesoderm. The lateral plate mesoderm (blue part of the mesoderm in fig. 1) then divides into two layers to form a space known as the embryonic coelom. Individually, the layers are known as the splanchnopleure and the somatopleure. The splanchnopleure (the inner side of the blue part in fig. 1) is in contact with and connected to the underlying endoderm layer (yellow) and later 
Avijgan and Avijgan: Meraque or Triple Energizer (San Jiao): Actual or Virtual Organ in Traditional Medicine - A Hypothetical Viewpoint

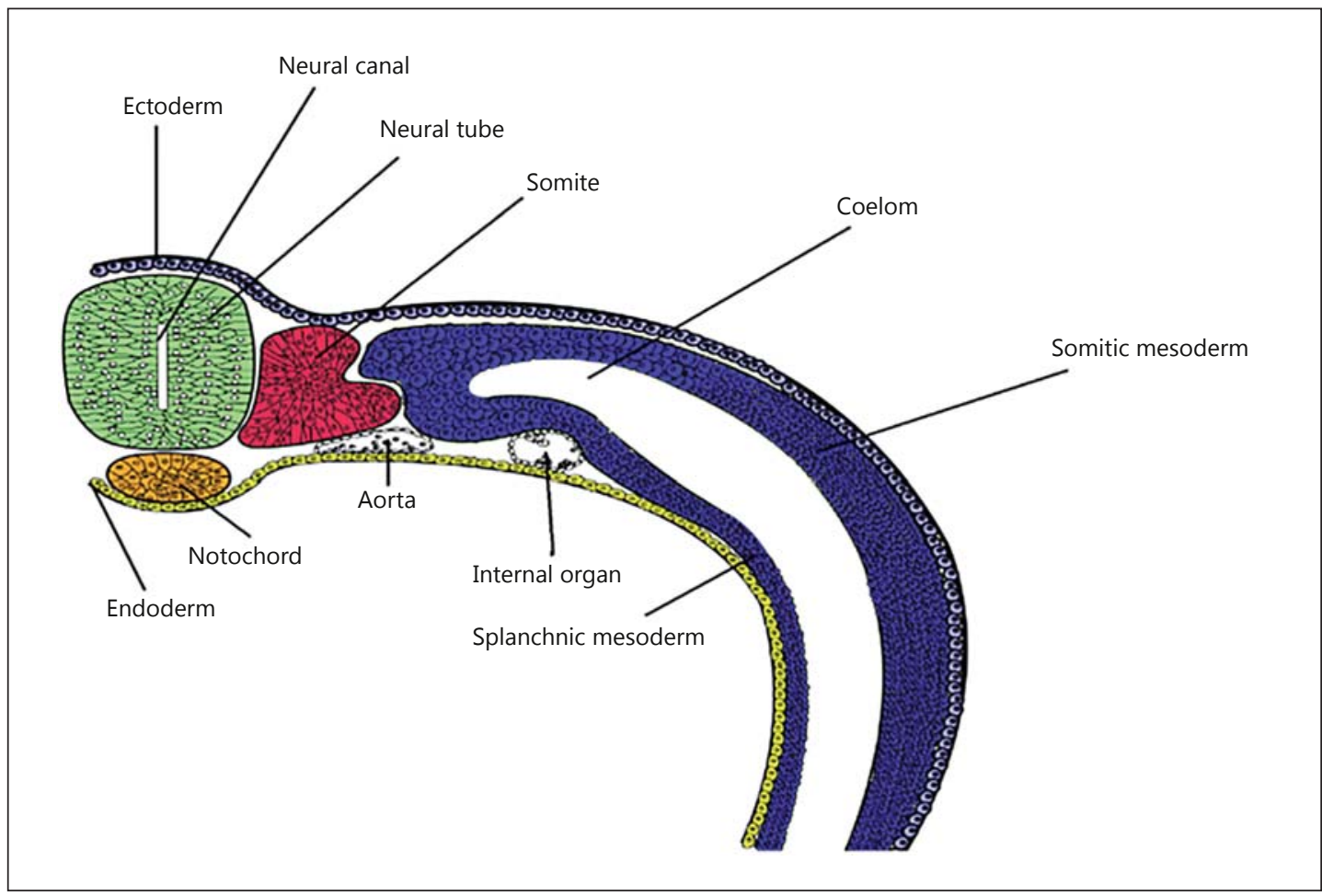

Fig. 1. Schematic illustration of the three layers of the embryo and location of the coelom (intraembryonic coelom).

is associated with primitive heart formation, the viscera and the serous membrane enveloping the internal organs as a sheet, capsule or stroma [11]. The somatopleure (the outer side of the blue part in fig. 1) is in contact with the ectoderm and later becomes the serous membrane which is in contact with the body wall (serosal mesoderm or subcutaneous tissue) [11].

The embryonic mesoderm is the origin of the mesenchyme, which is called mesenchymal connective tissue too. 'Mesenchymal' is basically a term used to describe the morphology of a kind of embryonic cell which remains a stem cell during its lifetime. These cells can be transformed for regenerating damaged tissue of the lymphatic system, circulatory system, connective tissue, etc. This meshwork is identified as a matrix material that contains reticular fibers and undifferentiated mesenchymal cells. The mesenchymal cells can easily migrate (unlike epithelial cells, which lack mobility and are adhered or organized into sheets). This mesodermal layer, in addition to the mesenchyme (connective tissue), forms the mesothelium, nonepithelial blood cells as well as coelomocytes. The mesothelium lines coeloms and forms the muscles, septa (cross-wise partitions) and mesenteries (length-wise partitions) [12].

The coelom (intraembryonic coelom) is like a bag with two layers: one located on the dorsal side and the other one located on the ventral side. Thus, some tissues such as the pancreas and the sexual organs, urinary bladder and kidney are created by the somatopleuric layer and located in the dorsal wall of the embryo, and some other organs such as the liver, gall bladder, small intestine and large intestine, which are created by the splanchnopleuric layer, are located in connection with the peritoneal serous and near-central cavity of the body [11]. 


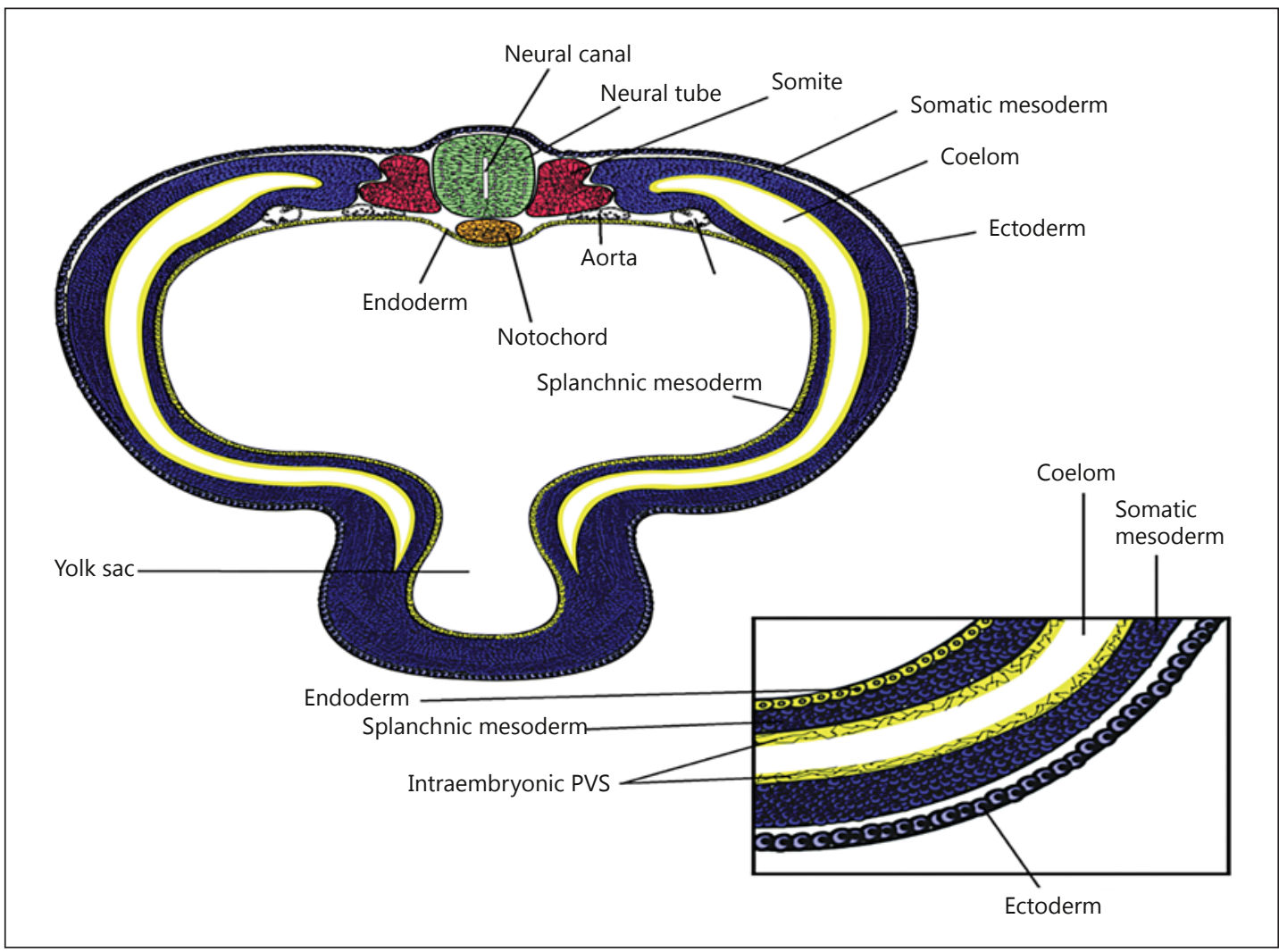

Fig. 2. Schematic illustration of the embryo. Inset Magnified part showing the layer of the theoretical PVS (yellow) in close contact with the somatic and splanchnic mesoderm $[5,7,8]$.

The intraembryonic coelom is a fluid-filled cavity in some animals in whom loss of the intraembryonic coelom is associated with a body size reduction. The coelom may absorb shocks like a hydrostatic device. In mammals, the intraembryonic coelom forms the pericardium, pleura and peritoneum $[5,11]$ (fig. 2).

\section{Cavities of the Body Originating from the Intraembryonic Coelom}

During the fourth week of the fetal life, the intraembryonic coelom is divided into the cavity of the pericardium, pleura and the peritoneum [11]. The first partition, the septum transversum, is the source of the future diaphragm and hepatic bud. The ventral mesentery or anterior part divides the intraembryonic coelom into primary peritoneal and pericardial cavities which are connected by the pericardioperitoneal duct. Pleuropericardial folds (buds) appear in the lateral side of the primitive pericardial cavity and form a wall dividing the cavity into pericardial and pleural cavities, by which the pleural wall closes the pericardioperitoneal duct [11]. Figure 2 shows the intraembryonic coelom as a cavity surrounded by ectoderm and endoderm, and illustrates that its mesoderm will subsequently form the organs of the ectoderm and endoderm.

\section{Historical Evidence for the Compatibility between the Intraembryonic Coelom and the} Meraque/TE/SJ

Is the intraembryonic coelom the cavity of the Meraque or TE/SJ? On the one hand, it has been said in ITM and TCM that the Meraque and TE/SJ are Fu and hollow organs, respectively; 
on the other hand, it has been shown that the intraembryonic coelom is a hollow organ too. This space is the most important in the human body. Taking into consideration that one layer is the inner layer (covering the endoderm) and another layer is the outer layer (under the ectoderm), it is clear that a collapsed space is between these two layers, which may be the TE/SJ or Meraque. It is suggested that the intraembryonic coelom, Meraque and TE/SJ are the same space, but it is necessary to understand this concept thoroughly.

For better understanding the Meraque/TE/SJ, one must look at embryology from another viewpoint. Based on our theory, the intraembryonic coelom is better to be called pericardial cavity, which is divided into the cavities of the pericardium, the pleura and the peritoneum. The ventral mesentery or anterior part of the septum transversum separates the primary peritoneal cavity from the pericardial cavity (intraembryonic coelom), which are connected by the pericardioperitoneal channel or duct. Then, pleural folds (buds) appear in the lateral side of the pericardial cavity and divide this cavity into the final pericardial and pleural cavities so that the pericardioperitoneal duct can be closed.

As has been shown, the pericardium is known as a Yin (Zang) organ in TCM. Moreover, it has been suggested that the intraembryonic coelom (pericardial cavity) is the origin of the peritoneal (at first) and then the pleural cavities, which will be transformed into the space of the Meraque/TE/SJ. The pericardium is the only Yin organ which has a formed cavity, but other Yin organs (except the Heart) such as the Liver, Lung and Spleen are formed and originate from the margin of this cavity (by an invagination process). By this hypothesis, the meaning of the paired organs of the pericardium/TE (as an imaginary and mysterious concept in TCM) is clarified. The pericardium (PC acupuncture channel) is a Yin organ, from which the TE/SJ as a Yang organ originates.

In other words, the cavity of the pericardium (intraembryonic coelom), which is a Yin organ (Zang, meaning 'storage site'), is the origin of (but not originating from) the TE/SJ, some part of which is later transformed into some other Yin organs (Lung, Liver and Spleen [11]) as well as other Fu organs including the TE/SJ. In this way, the concept of a distribution of the SJ or TE all over the human body becomes clear. In ITM, the Meraque has a similar role, which may be compatible with that of the TE/SJ.

Based on the suggested evidence, the extraembryonic PVS in hens' eggs $[6,7,13]$, even before incubation [13] and simultaneous with gastrulation, must enter and ingress into the embryo for the formation of the intraembryonic PVS [8]. By further development, it may be divided into two branches, one with the parietal mesoderm (the layer beneath the ectoderm or somatopleuric layer) and another along with the visceral mesoderm (the layer that surrounds the endoderm, which is splanchnopleuric), both of them forming a holistic network of an interconnected PVS. In other words, the extraembryonic and intraembryonic PVS together make a single and holistic PVS network $[8,13]$. This hypothesis is based on the belief that the PVS network found inside and outside of chicken embryos [6] may possibly exist in the human zygote or embryo too. This claim needs to be substantiated, which, however, is definitely prohibited by ethical concerns [14].

The theory of a PVS (intraembryonic or extraembryonic) [8] in the human embryo suggests that the extraembryonic PVS ingresses during gastrulation and is associated with the mesoderm; moreover, together with the mesoderm, it will be divided into two layers, the somatic and the visceral mesoderm, which later forms the serous membranes of the internal organs and subcutaneous connective tissues (associated with the ectoderm) or the skin [8] (fig. 2).

This hypothesis states that during gastrulation, the PVS, in its extraembryonic form, ingresses for (1) the formation and differentiation of the embryonic layer and (2) stimulation of the formation and/or appearance of the mesoderm. According to this theory, when the extraembryonic PVS enters into the embryo and is accompanied by the mesoderm, it will be 
divided into two distinct layers which surround the space of the intraembryonic coelom (pericardial cavity and TE/SJ/Meraque) [8] (fig. 2, inset).

Thus, our claim can lead to the idea that the Meraque/TE/SJ is a Fu organ from which the major organs of ITM and TCM are derived. In ITM, the major organs are called organs of reiseh, including the Brain, Heart and Liver, whereas in TCM, they are Yin and Yang organs. Based on this concept, the pericardial cavity (intraembryonic coelom) is the origin of the TE/SJ/ Meraque, which is the source of some major organs and is a connecting pathway between them. This concept may also be a basis for fasciology [5].

Among five organs, the Kidney is exceptional. The Kidney organ originates from the mesodermal part in the retroperitoneal space and is the source of Ming Men Fire or Minister Fire. It is located on the back side of the body, where the UB acupuncture channel is located. This may explain and justify why the Back Shu points are on the UB channel and are also in close contact with the Kidney. The Kidney is the source of the Minister Fire and by the Back Shu points can stimulate the Fire or Yang of organs (unpublished data).

In addition, the coelom or SJ is like a bag of which the anterior wall is under the skin of the abdominal wall (and presenting the Meraque in ITM); it is in close contact with Ren Mai, the end part of the Kidney, Spleen and Liver channels and the starting part of the Heart, Lung and Pericardium channels. This side of the SJ/TE or Meraque can tonify the Yin of organs.

In summary, it is clear why the SJ/TE or Meraque is in close contact with all Yin organs (in the anterior wall of the intraembryonic coelom) and also Yang organs (which originate from it) and therefore a holistic network of the human body. Among the Yin organs, the Heart and Kidney are exceptional organs which need a more detailed explanation (unpublished data).

\section{Histology of the Meraque/TE/SJ}

The human body is made up of four types of tissue, of which connective tissue is one; the other three types are epithelia, muscles and nerves. Connective tissue is a space-filling tissue. The vascular tissues of the blood and lymph vessels are part of this tissue. Connective tissue is of two main types, loose and dense. The dense type may be specialized to make specific tissue such as muscle fascia or liver or spleen capsule, but the loose type is a space-filling tissue which fills all the space between organs. Loose connective tissue is made of collagen fibers, elastin fibers, macrophages, fibroblasts, adipocytes, capillaries and the space between them. This space is filled by the extracellular matrix (ECM). The ECM is an amorphous gelatinous substance which includes glycosaminoglycans (GAGs). There are four types of GAG, of which hyaluronic acid is the simplest, because it is a nonsulfated sugar; the other three types are sulfated sugars.

All types of GAG except hyaluronic acid attach to a core protein to make proteoglycans. Proteoglycans are very heterogeneous, and they can form different sizes of pores for different sizes of molecules to be regulated when they are moving through the matrix. These large substances compose about $10 \%$ of the ECM, but $90 \%$ of this space is filled with water which is absorbed by proteoglycans like a sponge. In this way, the ECM works as a compressive force absorbent [15].

This structure, which may be called serous membrane, is formed from a secretory epithelial layer and a connective tissue layer underneath. The epithelial layer, known as the mesothelium, consists of a single layer of avascular flat nucleated cells (simple squamous epithelium) which produce the lubricating serous fluid. This fluid has a consistency similar to thin mucus. These cells are firmly attached to the underlying connective tissue. The connective tissue layer provides the blood vessels and nerves for the overlying secretory cells, and also acts as the binding layer which allows the whole serous membrane to adhere to the organs and other structures (this can be loose connective tissue). 
Avijgan and Avijgan: Meraque or Triple Energizer (San Jiao): Actual or Virtual Organ in Traditional Medicine - A Hypothetical Viewpoint

Fig. 3. Stereomicroscopic images of Bonghan ducts (PV) and corpuscles on the surface of a rabbit's internal organs. a Bonghan duct (PV; arrow) on the large intestinal surface (LI). This is an intact duct, a semitransparent, freely movable tissue structure. b Bonghan duct (PV; arrow) after methylene blue staining. Reprinted with permission from Dr. Kwang-Sup Soh [6].
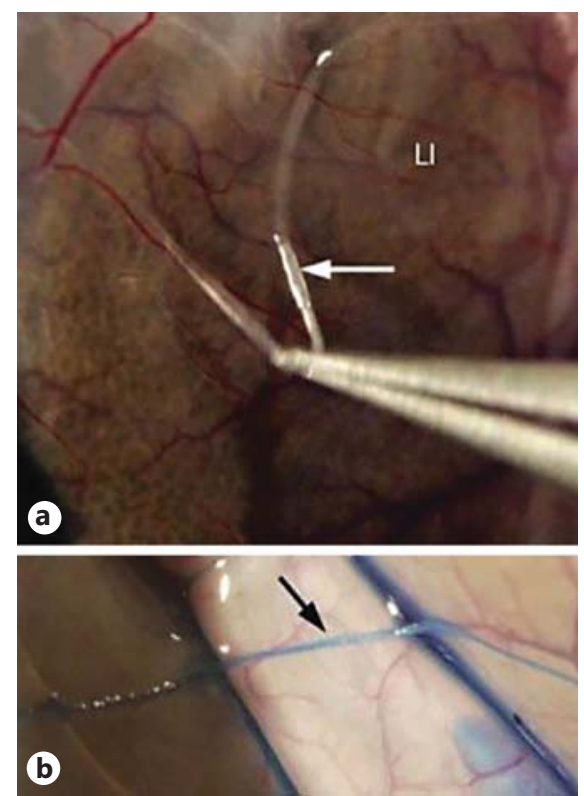

In the Tebbe Akbari, which is a Persian version of the Asbab va Alaem written 820 years ago, it has been indicated (chapter 20, p. 924) that in the abdominal wall there is a skin and membrane which is called Meraque. The inner side of the abdominal cavity consists of muscles and two more membranes, one of them the inner membrane, called sarb (in contact with the stomach and intestine), and the other one the outer membrane, called sefagh (meaning the 'continuous of jaufs'). There is an upper jauf (maybe synonymous with the upper SJ) and a lower jauf (maybe synonymous with the middle and lower SJ). Thus, in this sense, the Meraque is an exterior membrane which includes two other membranes of inner and outer membranes. In other words, the Meraque is an expanded membrane which includes two limited membranes. In this sense, the Meraque membrane in ITM may be similar to the SJ space of TCM.

In our previous study, we reported on the PVS [5], which must be passing along the loose connective tissue to connect all cells and organs in a holistic network. Figure 3 shows the loose attachment of the PVS to the connective tissue (most probably loose connective tissue). The serous membrane where the PVS [8] is passing can be considered as the Meraque/TE/ SJ.

Given that in the ECM there are many spaces which are filled with water, the meaning of two other traditional terms becomes clear. In ITM there are tajvif, spaces inside the organs, and in TCM there is Cou Li, which is like a groove or space; both of them are places where nutrients are exchanged. In other words, if connective tissue can be considered as a holistic tissue that can connect the exterior part of the body as the Meraque (under the skin and among muscles) with the interior part of the body (sarb and sefagh), this can explain the effect of acupuncture points on internal organs throughout the TE/SJ space, which is the pathway of the PVS $[7,8]$ and a holistic organ in the body (fig. 3), being the same as the Meraque/TE/ SJ, Cou Li or tajvif.

Serous cavities, made by the intraembryonic coelom, are hollow spaces in the margin of the intraembryonic coelom, and they are like small cavities enveloped by the serous membrane. Based on this idea, the body is composed of thousands and thousands of cavities.

In early fetal life, the visceral organs are developed by the process of invagination into this space and finally are enveloped by the double layer of serous membranes, one attached to the organs as a visceral layer and the other with a collapsed space as a parietal layer (fig. 4). 
Fig. 4. The process of budding and invagination of the internal organs during organogenesis in the fetal period.

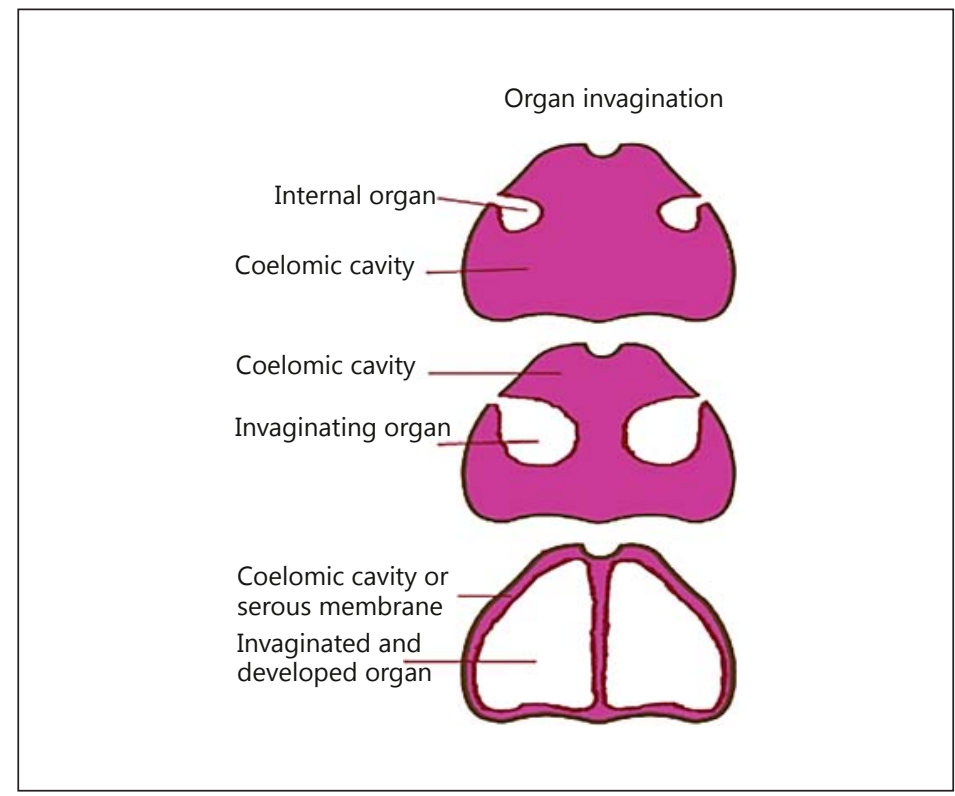

By this process, the internal organs (which originate from the mesodermal layer of the splanchnopleure) bud into the intraembryonic coelom and, by developing this process, finally are established and wrapped by connective tissue (of the mesoderm), which is the same as the TE/SJ or Meraque (pink parts in fig. 4).

The Meraque/TE/SJ can be viewed from three perspectives or three aspects: first, as a system of waterways (nutrients); second, as cavities (spaces of tajvif and Cou Li), and, third, as a three-fold space of the body (SJ). In other words, the intraembryonic coelom forms a network of serous membranes (cavities) including the mesothelioma and mesenchyme, so that the developing organs will invaginate into the cavity of the intraembryonic coelom for final generation; at the end of the process of fetal development there are thousands of disappeared cavities between the organs (a collection of small or large cavities), where the Meraque/TE/SJ is suggested to be.

\section{Meaning of SJ according to Opinions in TCM}

Based on our previous report, TCM is based on Qi and Qi movement, and the SJ is one of the main ways for the passage of Qi and Yang Qi. If Zang is the storage (source) site in TCM, $\mathrm{Fu}$ denotes the passage site [16]. First of all, it is better to differentiate between SJ channel and SJ space. The SJ channel is a channel like the other 13 acupuncture channels. But the SJ space denotes the space which is different from the SJ channel.

The Nei Jing, a famous Chinese medical doctor of the late Qing dynasty (Rong-chan Tang, AD 1862-1918), Hao-gu Wang, a famous Chinese medical doctor of the Yuan dynasty, and the authors of a textbook from the Beijing Chinese Medical Continuing Education Center describe the SJ space (not the SJ channel), and this is a little confusing [16]. However, Dong-yuan Li (AD 1180-1251), a famous Chinese medical doctor of the Yuan dynasty, had unique opinions which have some similarities to our theory. He said that 'the upper, middle, and lower San Jiao connect to be one Qi and to defend the human body', and that the 'San Jiao is not a real Fu with no structure but having a function'. The upper SJ contains the air of all the cleft between the thorax and the diaphragm, with exception of the lung lobes. The middle SJ contains the air of all the cleft within the abdomen with exception of the spleen, kidney, pancreas and intestines. The lower SJ contains the air of all the cleft with exception of the large intestines and urinary bladder. 
Avijgan and Avijgan: Meraque or Triple Energizer (San Jiao): Actual or Virtual Organ in Traditional Medicine - A Hypothetical Viewpoint

Fig. 5. a-c Connective tissue images are marked and their 3D structure is rendered. $\mathbf{d}$ When the fascial connective tissue of the whole body was marked and its 3D structure was rebuilt, a complete fascial network was observed. Reprinted with permission from Prof. Yuan Lin and Dr. Bai Yu [21].

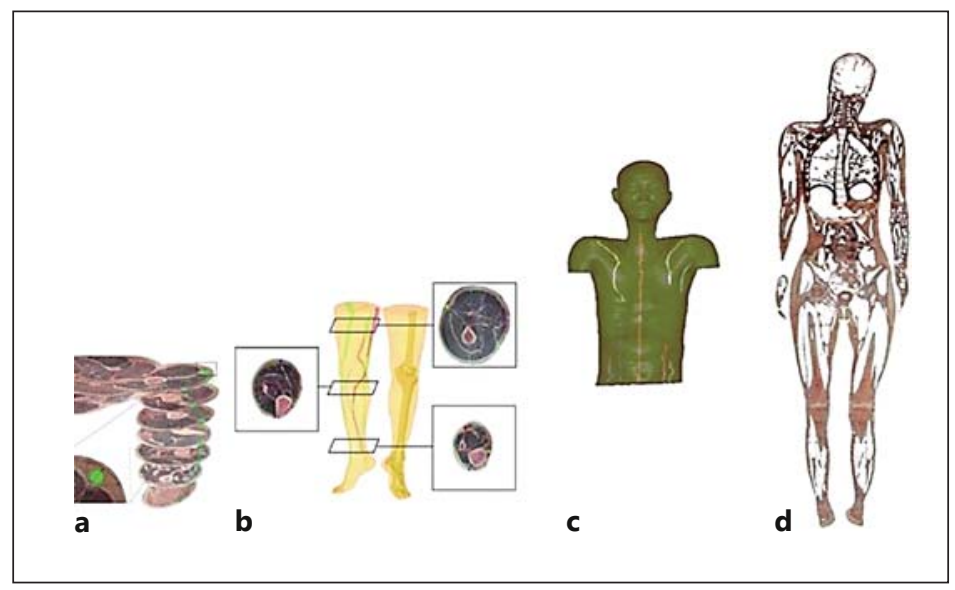

After a comparison between the human body and nature, he claims that as the space between heaven and earth is not useless, the $\mathrm{SJ}$ is like the air of this space and contains the Qi inside the human body. This part of the theory of Dong-yuan Li is similar to our theory indicating that the PVS is passing along the space inside the loose connective tissue and is a passageway for the produced Qi [16]. But in the Nei Jing, the SJ has two meanings, sometimes as $\mathrm{Qi}$ and sometimes as a location. The location has been variously described in differing theories and ideas. It is better to describe the location of the SJ in the space of the loose connective tissue, where it is a passageway for the Qi, so that this theory may cover both concepts of the Nei Jing (Qi and location). Given that tajvif and Cou Li are spaces inside the organs, it becomes understandable that the SJ (and also the Meraque) contains the air of all the cleft.

In opposition to the claims of Dong-yuan Li, who said that 'the San Jiao is not the primary Fu' and that 'there is no shape at all but it is useful' [16], our theory suggests that the SJ/ Meraque originates from the pericardial cavity (intraembryonic coelom) and is the origin of the peritoneal and pleural cavities (the upper and the lower jauf, respectively) so that it extends as the fetus and PVS extend. Therefore, it may be suggested to be the primary Fu organ which is developed and is the origin of other Fu organs such as the Stomach, Small Intestine, Large Intestine, Gall Bladder and even Urinary Bladder as well as the SJ channel (unpublished data).

In the theory of Qi as air in the space of the human body, the following question is asked: 'Why, after excising or removing an internal organ to release the Qi, does it not cause damage instead of dramatically curing diseases?' The author replies that 'most Qi can aggregate and also disperse; after Qi is released, it will be produced again immediately' [16]. Based on our theory, in the case of surgical removal of an organ and releasing the space of loose connective tissue, there is no free Qi (like air) to be released, but the Qi moves inside the PVS and is responsible for tissue repair after surgery and has a physical property which is produced in the PVS [7].

In the Nei Jing, with regard to the Qi and SJ, three types of Qi are defined: strong primary Qi, internal primary Qi and Shao Yang Qi. In practice, the definition and direction of all types of Qi may be compatible with our theory that there is a unique space of SJ including the PVS [7] in which the produced Qi is dispersed in all directions of the human body, i.e. to the top, bottom, right, left, inside and outside, and which covers all definitions of the direction and movement of Qi.

Recently, by presenting the Virtual Chinese Human Male No. 1 (VCH-M1) and Virtual Chinese Human Female No. 1 (VCH-F1), scientific evidence for the SJ as a real space has emerged and suggests that it is distributed along connective tissue [17-21] (fig. 5). 
In this amazing report, after the exclusion of muscle and other tissues, we propose that the connective tissue is the location of the meridians and points, and we suggest that they are dependent on the SJ/Meraque space.

\section{Conclusions}

All serous membranes in the human body are made of mesoderm in the trilaminar embryo. The intraembryonic coelom (pericardial cavity) can be used as a cavity within the body which is covered by the serous membranes originating from the somatopleure and splanchnopleure. This cavity is divided, during fetal development, into two serous cavities (pleural and peritoneal cavities) which latter are the origin of many different organs in the human body. These serous cavities indicate the meaningfulness of the concept of the Meraque in ITM and the TE/SJ in TCM, which is spread among the organs of the human body.

Surely, the main limitation to research on the subject of the Meraque/TE/SJ is an ethical concern regarding research on human embryos. But with our hypothesis, the intraembryonic coelom can generally be attributed to the Meraque/TE/SJ. In other words, the Meraque/TE/ SJ can be interpreted as and explained by the intraembryonic coelom, and by tracing it from the first day of conception to the final stages of fetal development, an invisible space (coelom) can clearly be seen between the anatomical organs which is suggested to be the Meraque/TE/ SJ.

\section{Suggestions}

The TE is probably the most widely discussed topic in Chinese medicine, and, over the centuries, there have been many different theories about and explanations of its nature.

There are at least six different ways of looking at the SJ/TE, as listed below:

1 as a system of waterways (in loose connective tissue);

2 as a system of cavities (e.g. Cou Li or tajvif);

3 as a three-fold division of the body (pericardial, pleural and peritoneal);

4 with regard to the relationship between the pericardium and the TE (which have the same origin);

5 as the activator of the Yuan Qi (PVS), and

6 as governing movement of the Qi (produced Qi inside the PVS).

The other features of the SJ and Meraque must be explained by this theory, which, however, has to be discussed at another opportunity.

\section{Acknowledgment}

We thank Mrs. Fatemeh Abdollahi for her kindness and helpful editing of this paper.

\section{References}

1 Bahrami M, Nazem I, Mastri Farahani R, Kamali Nezhad M, Keshavarz M: Ghalaz vessel (in Persian). J Islam Iran Trad Med 2012;3:143-147.

2 Huang Ti Nei Jing Su Wen: The Yellow Emperor's Classic of Internal Medicine-Simple Questions. Beijing, People's Health Publishing House, 1979 [1st publ ca 100 BC], p 59.

3 Zhong Yi Li Dai Yi Lun Xuan: Medicine Treasure; cited in Wang Xin Hua Selected Historical Theories of Chinese Medicine. Nanjing, Jiangsu Scientific Publishing House, 1983, p 2.

4 Ling Shu Jing: Spiritual Axis. Beijing, People's Health Publishing House, 1981 [1st publ ca 100 BC], p 71. 
5 Avijgan M, Avijgan M: Triple Burner or San Jiao as a real space or false space of human body. 2nd International Conference and Exhibition on Traditional and Alternative Medicine, Beijing, China, August 25-26, 2014. http://omicsgroup.com/tradiitonal-alternative-medicine-conference-2014/index.php (accessed May 15, 2015).

6 Lee S-Y, Lee B-C, Soh K-S, Jhon G-J: Development of the putative primo Vascular System before the formation of vitelline vessel in chicken embryo; in Soh K-S, et al (eds): The Primo Vascular System: Its Role in Cancer and Regeneration. London, Springer, 2011, pp 77-82.

7 Avijgan M, Avijgan M: Can the Primo Vascular System (Bong Han duct system) be a basic concept for Qi production? Int J Integr Med 2013;1:20.

8 Avijgan M, Avijgan M: Does the Primo Vascular System Originate from the Polar Body? Integr Med Int 2014;1: 108-118.

$\checkmark 9$ Onegawa A, Funayama N, Ueno N, Takahashi Y: Mesodermal subdivision along the mediolateral axis in chicken controlled by different concentrations of BMP-4. Development 1997;124:1975-1984.

10 Kimelman D, Bjornson C: Vertebrate mesoderm induction: from frogs to mice; in Stern CD (ed): Gastrulation: From Cells to Embryo. Long Island, CSHL Press, 2004, p 363.

11 Larsen WJ: Essentials of Human Embryology. Edinburgh, Churchill Livingstone, 1998.

12 Ruppert EE, Fox RS, Barnes RB: Introduction to bilateria; in Ruppert EE, Fox RS, Barnes RB (eds): Invertebrate Zoology, ed 7. Belmont, Brooks/Cole, 2004, pp 217-218.

13 Lee SY: Master's thesis, Seoul National University, 2010.

14 June PL: Embryonic stem cell research. Pediatrics 2002;109:990-991.

15 http://www.histology.leeds.ac.uk/tissue_types/connective/connective_groundS.php (accessed March 25, 2015).

16 Chen D-F: Correct errors of San Jiao. J Accord Integr Med 2009;5:138-144. http://www.accordinstitute. org/2009_5_3_correct_errors_of_san_jiao.htm.

17 Yuan L, Zhong SZ: Human auto-testing and modulating system - anatomic basis involved in meridianal system (in Chinese). Tianjin J Trad Chin Med 2004;21:356-359.

18 Yuan L, Tang L, Huang WH, et al: Construction of dataset for virtual Chinese male No. 1. Di Yi Jun Yi Da Xue Xue Bao 2003;23:520-523.

19 Wang CL, Bian J, Yuan L, et al: A digital study on the morphologic comparability between meridians and rebuild fascia lines in the VCH-Ml's lower limb (in Chinese). Acta Anatomica Sinica 2008;39:219-222.

20 Wang CL, Wu JP, Wang J, et al: An interpretation on the essence of meridians and acupuncture mechanism from fasciology view (in Chinese). Chin J Basic Med Trad Chin Med 2008;14:312-314.

21 Lin Y,Yu B: Anatomical discovery of meridians and collaterals (poster). http://www.fasciacongress.org/2009/ abstract_pdf/posters/J_052\%20\%20\%20Anatomical\%20Discovery\%20of and\%20Collaterals\%5B1\%5D.pdf. 